\title{
In vitro generation of mature midbrain-type dopamine neurons by adjusting exogenous Nurr1 and Foxa2 expressions to their physiologic patterns
}

\begin{abstract}
Taeho Kim ${ }^{1,2,4}$, Jae-Jin Song ${ }^{1,2,4}$, Lesly Puspita ${ }^{3}$, Parvin Valiulahi ${ }^{3}$, Jae-won Shim ${ }^{3}$ and Sang-Hun Lee ${ }^{1,2}$
Developmental information aids stem cell biologists in producing tissue-specific cells. Recapitulation of the developmental profile of a specific cell type in an in vitro stem cell system provides a strategy for manipulating cell-fate choice during the differentiation process. Nurr1 and Foxa2 are potential candidates for genetic engineering to generate midbrain-type dopamine (DA) neurons for experimental and therapeutic applications in Parkinson's disease (PD), as forced expression of these genes in neural stem/precursor cells (NPCs) yields cells with a complete battery of midbrain DA neuron-specific genes. However, simple overexpression without considering their expression pattern in the developing midbrain tends to generate DA cells without adequate neuronal maturation and long-term maintenance of their phenotype in vitro and in vivo after transplantation. We here show that the physiological levels and timing of Nurr1 and Foxa2 expression can be replicated in NPCs by choosing the right vectors and promoters. Controlled expression combined with a strategy for transgene expression maintenance induced generation of fully mature midbrain-type DA neurons. These findings demonstrate the feasibility of cellular engineering for artificial cell-fate specification.
\end{abstract}

Experimental \& Molecular Medicine (2017) 49, e300; doi:10.1038/emm.2016.163; published online 10 March 2017

\section{INTRODUCTION}

In vitro production of functional tissue-specific cells is a critical issue in stem cell biology for both experimental and clinical applications. The midbrain dopamine $(\mathrm{mDA})$ neuron is of particular interest because of its significant physiological and clinical implications. Parkinson's disease (PD), characterized by progressive loss of $\mathrm{mDA}$ neurons in the midbrain substantia nigra $(\mathrm{SN}),{ }^{1,2}$ is the only neurodegenerative disease with a clinical history of cell transplantation using human fetal midbrains $s^{3,4}$ and thus is regarded as a prime target for cell therapeutic approaches. ${ }^{5}$ Neural stem/precursor cells (NPCs) cultured in vitro offer an alternative source for application of stem cells that overcomes the ethical and logistical problems raised by utilizing fetal midbrain tissue. Before establishing stable and functional cell transplantation methods in patients, animal modeling studies are fundamentally necessary. NPCs taken from the ventral midbrain (VM) of rat embryos are one of the potential cell source to obtain for transplantation studies because they naturally develop into $\mathrm{mDA}$ neurons. However, efficient DA neuron differentiation from VM-derived NPCs is limited to short-term expanded cultures derived from early embryonic days. ${ }^{6,7}$ Manipulation of the genes involved in $\mathrm{mDA}$ neuron development to direct non-midbrain NPC into midbrain-type DA neurons is a potential strategy to overcome this limitation.

Forkhead family of winged-helix transcription factor 2 (Foxa2; also known as HNF3 $\beta$ ) is among the earliest developmental transcription factors expressed in embryonic VM. It acts as a master regulator for $\mathrm{mDA}$ neuron development by inducing expression of a battery of genes that, in turn, regulate mDA neuron specification. ${ }^{8-12}$ Nuclear receptor-related factor 1 (Nurr1; also known as NR4A2) is a downstream factor expressed at later developmental stages and acts as a critical transcription factor to induce mDA phenotype gene

\footnotetext{
${ }^{1}$ Department of Biochemistry and Molecular Biology, College of Medicine, Hanyang University, Seoul, Korea; ${ }^{2}$ Graduate School of Biomedical Science and Engineering, Hanyang University, Seoul, Korea and ${ }^{3}$ Soonchunhyang Institute of Medi-bio Science (SIMS), Soonchunhyang University, Cheonan-si, Korea ${ }^{4}$ These authors contributed equally to this work.

Correspondence: Dr J-w Shim, Soonchunhyang Institute of Medi-bio Science (SIMS), Soonchunhyang University, 25, Bongjeong-ro, Dongnam-gu, Cheonan-si 31151, Korea.

E-mail: shimj@sch.ac.kr

or Dr S-H Lee, Department of Biochemistry and Molecular Biology, College of Medicine, Hanyang University, 222, Wangsimni-ro, Seongdong-gu, Seoul 04763, Korea.

E-mail: leesh@hanyang.ac.kr

Received 11 June 2016; revised 24 September 2016; accepted 9 November 2016
} 
expression. ${ }^{13-15}$ Foxa2 expression continues at later developmental stages, and it interacts with Nurrl in a feed-forward manner to induce mDA neuron development, ${ }^{9,16}$ indicating that both Foxa2 and Nurr1 are strong candidates to be engineered in cultured NPCs for midbrain-type DA neuron generation in vitro. Indeed, combined expression of Nurr1 and Foxa2 drives non-midbrain NPCs to differentiate into DA cells expressing the complete sets of DA phenotype and midbrainspecific genes. However, forced Nurr1 and Foxa2 expression frequently generates non-neuronal or immature DA cells. ${ }^{17-22}$ Furthermore, DA neuronal phenotypes and functions acquired by forced gene expression were not stable over long time periods. Consequently, transplantation of engineered NPCs resulted in poor DA neuron engraftment and functional recovery in PD animal models.

In this study, we aimed to improve Nurr1+Foxa2 engineering to yield mature and functional midbrain-type DA neurons with stable phenotypes that can be maintained for a long period of time. We recently showed that expression levels and timing of exogenous gene induction that mimic physiological patterns may be important to yield mature and functional tissue-specific cells from cultured stem/precursor cells. ${ }^{17}$ Thus, to achieve maturity and functionality of the resulting midbrain-type DA neurons, we chose appropriate expression vectors and promoters to induce the level and timing of exogenous Nurr1 and Foxa2 expression in cultured NPCs such that they resemble those in the developing VM. Sustained Nurr1 and Foxa2 exogenous expression levels are important in this strategy, as both Nurr1 and Foxa2 are physiologically expressed in $\mathrm{mDA}$ neurons in the adult midbrain and play critical roles in cell survival, function and phenotype maintenance. ${ }^{23-28}$ However, we observed that exogenous expression induced by most of the tested vector systems was easily and selectively silenced in the DA neuronal population. Therefore, we further included a strategy to prevent silencing of ectopic gene expression by choosing appropriate tools to maintain stable exogenous gene expression. This study provides a strategy to acquire a specific type of neurons using artificial gene manipulation that is physiologically relevant to in vivo conditions.

\section{MATERIALS AND METHODS}

\section{NPCs culture}

NPCs were isolated and cultured from VMs or cortices of rat embryos at embryonic days 12 and 14 (E12 and E14). Cells were plated on 6 or $10 \mathrm{~cm}$ poly-L-ornithine $\left(15 \mu \mathrm{g} \mathrm{ml}^{-1}\right.$, Sigma, St Louis, MO, USA)/ fibronectin ( $1 \mu \mathrm{g} \mathrm{ml}^{-1}$, Sigma) pre-coated dishes (Corning, NY, USA) and allowed to proliferate in the presence of basic fibroblast growth factor (bFGF, $20 \mathrm{ng} \mathrm{ml}^{-1}$, R\&D Systems, Minneapolis, MN, USA) in serum-free N2 medium. ${ }^{29}$ To gain a homogenous population of NPCs, the expanded cells were passaged by dissociating cells into single cells and re-plating them onto prepared PLO/FN pre-coated coverslips (12-mm diameter; marienfeld GmbH \& Co., KG, Lauda-Konigshofen, Germany). Cell proliferation was maintained upon reaching 50-60\% cell confluency in the presence of bFGF (usually for 1-2 days) before differentiation was induced by the elimination of the mitogen. bFGF elimination in E12 VM NPC culture ${ }^{30}$ directed the NPCs to differentiate without prior passaging. Cultures were incubated at $37^{\circ}$ $\mathrm{C}$ in a $5 \% \mathrm{CO}_{2}$ atmosphere. In certain experiments, $0.5 \mathrm{~mm}$ cyclic AMP (cAMP; dibutyryl-cAMP, Sigma) was added to the medium.

\section{Immunocytochemistry}

Brain tissue and cells were fixed in $4 \%$ paraformaldehyde (PFA). Fixed tissues were blocked in $0.3 \%$ Triton X-100 with $1 \%$ bovine serum albumin (BSA) for $40 \mathrm{~min}$ and incubated with primary antibodies overnight at $4{ }^{\circ} \mathrm{C}$. The following primary antibodies were used: rabbit anti-green fluorescence protein (GFP, 1:2000, Invitrogen, Eugene, OR, USA), mouse anti- $\beta$-tubulin type III (Tuj1, 1:500, Covance, Emeryville, CA, USA), mouse anti-human nerve growth factor IB (NGFI-B) $\beta$ (Nurr1, 1:1500, R\&D Systems), goat anti-Foxa2 (1:500, Santa Cruz Biotechnology, Dallas, TX, USA), rabbit anti-tyrosine hydroxylase (TH, 1:1000, Pel-Freez, Rogers, AR, USA), mouse anti-TH (1:1000, Sigma), mouse antiproliferating cell nuclear antigen (PCNA; 1:40, Millipore, MA, USA), mouse anti-microtubule-associated protein 2 (MAP2, 1:500, Sigma), rabbit anti-Pitx3 (1:200, Life Technologies, Waltham, MA, USA), rabbit anti-Vesicular monoamine transporter 2 (VMAT2; 1:500, Pel-freez), mouse anti-NeuN (1:200, mouse, Chemicon, Temecula, CA, USA), rat anti-dopamine transporter (DAT, 1:200, Abcam, Cambridge, MA, USA). Secondary antibodies tagged with Cy3 (Jackson Immunoresearch Laboratories, West Grove, PA, USA) and Alexa488 (Invitrogen) were used. Brain tissue and cells were mounted using VECTASHIELD with DAPI (Vector Laboratories, Burlingame, CA, USA). Images were obtained through the fluorescent microscopy and confocal microscopy (Leica PCS SP5, Leica, Heidelberg, Germany).

\section{Plasmid constructions and retroviral and lentiviral production and infection}

Retroviral vectors expressing Nurr1, Foxa2 or GFP under the control of retroviral long terminal repeat (LTR) promoter (pLTR) were constructed by inserting each cDNA fragment into the multi-cloning sites of the retroviral vector $(p C L)$ as described previously. ${ }^{20}$ The retroviral vectors were transfected into 293GPG packaging cells. Lentiviral vector expressing Nurr1, Foxa2, ca-PKA or GFP under the control of various promoters (CMV, CAG, PGK, EF1a, Ubiquitin) were cloned in the lentiviral backbone vector $(p C D H)$, and were transfected into H293T cells using packaging particles. Lipofectamine (Lipofectamine 2000, Invitrogen) was used for transfection, and the supernatant containing viral particles was harvested $72 \mathrm{~h}$ (retroviral particles) or $24 \mathrm{~h}$ (lentiviral particles) after incubation. NPCs were treated with viral supernatant for $2-4 \mathrm{~h}$ with polybrene $\left(1 \mu \mathrm{g} \mathrm{ml}{ }^{-1}\right.$, Sigma), cultured overnight in the presence of bFGF, and then induced to differentiate. Co-expression studies were carried out by infecting cells with mixtures of individually prepared virus (1:1). Viral titers were determined with a viral quantitation kit (QuickTiter Retrovirus Quantitation Kit and QuickTiter HIV Lentivirus Quantitation Kit, (Cell Biolabs, Inc., San Diego, CA, USA) and adjusted to $1 \times 10^{6}-10^{8}$ $\mathrm{TU}$ (transducing unit) $\mathrm{ml}^{-1}$. Titers of viruses expressing the reporter GFP, used in gene expression pattern analyses, were determined based on GFP expression in HEK293 cells using FACS. ${ }^{31}$

\section{DA measurement}

DA release assays were carried out in differentiated NPC cultures at differentiation day 15. Media incubated for $24 \mathrm{~h}$ were collected. DA level determination of the media was performed using an ELISA kit 
(BAE-5300, LDN). In addition, cultures were incubated in fresh N2 media containing $56 \mathrm{~mm} \mathrm{KCl} \mathrm{(evoked)} \mathrm{or} \mathrm{not} \mathrm{(basal} \mathrm{release)} \mathrm{for}$ $30 \mathrm{~min}$. Evoked DA release was calculated by subtracting the basal release from the $\mathrm{DA}$ level of the $\mathrm{KCl}$-containing medium.

\section{Cell toxicity assay}

NPCs derived from rat embryonic cortices at E14 were transduced with Nurr1 and Forxa2 as described above. After 13 days of in vitro differentiation, cultures were treated with $\mathrm{H}_{2} \mathrm{O}_{2} \quad(31.25-250 \mu \mathrm{M}$; Sigma-Aldrich) for $8 \mathrm{~h}$, and cell viability was determined by directly counting $\mathrm{TH}+$ cells.

\section{In vivo transplantation}

Male Sprague-Dawley rats weighing $220-250 \mathrm{~g}$ at the start of the experiment were used for all in vivo studies. Under pentobarbital anesthesia, $4 \mu \mathrm{l}$ of 6-hydroxydopamine (6-OHDA, $3 \mu \mathrm{g}^{-1} \mathrm{l}^{-1}$ in normal saline containing $0.2 \mathrm{mg} \mathrm{ml}^{-1}$ ascorbic acid (AA)) was injected stereotaxically into the $\mathrm{SN}$ and the median forebrain bundle (4.8 and $1.8 \mathrm{~mm}$ posterior to bregma; 1.5 and $1.8 \mathrm{~mm}$ lateral to midline; 8.2 and $8.0 \mathrm{~mm}$ ventral to the dura). The incisor bar was set at $3.5 \mathrm{~mm}$ below zero (interaural line). Two days after viral infection (in vitro differentiation day 1), cells were dissociated by trypsinization and suspended in normal saline. Animals were anesthetized with pentobarbital and placed in a stereotaxic frame. In each of eight animals, $3 \mu \mathrm{l}$ of the cell suspensions $\left(3 \mu \mathrm{l}, 2 \times 10^{5}\right.$ cells per $\mu \mathrm{l}$ in $\mathrm{N}_{2}$ with virus medium) was injected over a 5 - min period in the lesioned striatum at two sites coordinates in anteroposterior, mediolateral and dorsoventral relative to bregma and dura: (a) 0.07, $-0.30,0.55$; (b) $-0.10,-0.40,-0.50$; incisor bar set at $3.5 \mathrm{~mm}$ using a 24-gauge needle and KDS310 nano pump (KD Scientific Inc., Holliston, MA, USA). The needle was left in place for $3 \mathrm{~min}$ following completion of each injection. The rats received daily injections of cyclosporine A (10 $\mathrm{mg} \mathrm{kg}^{-1}$ i.p. injection) starting 1 day before grafting and continuing for 20 days.

\section{Histological procedures}

Cell transplanted animals were perfused transcardially with 4\% PFA. Rat embryos were fixed with $4 \%$ PFA for $20 \mathrm{~min}$. Adult brain tissue and embryos were immersed in 30\% sucrose in PBS overnight, frozen in Tissue-Tek (Sakura Finetek USA, Torrance, CA, USA) and sectioned at $40-\mu \mathrm{m}$ (adult brain) or $12-\mu \mathrm{m}$ (embryo) thickness on a freezing microtome (CM 1850, Leica). The slices were stained with antibodies as described above.

\section{Cell counting and statistical analysis}

Immunoreactive or DAPI-stained cells derived from at least three independent experiments. were randomly counted in 10-20 areas using an eyepiece grid. Data were expressed by mean \pm s.e.m. of three to five independent cultures. Statistical analysis was done using ANOVA with Tukey post hoc analysis (IBM SPSS Statistics; IBMCorp., NY, USA, http://www.IBM.com).

\section{RESULTS}

Gene expression patterns induced by retroviral and lentiviral vectors with various promoters in cultured NPCs

Combined Nurr1+Foxa2 expression in non-midbrain NPCs efficiently generates midbrain-type DA cells expressing a complete set of DA phenotype and midbrain-specific gene expression. ${ }^{8}$ However, simple overexpression of these genes often yields non-neuronal or immature midbrain-type DA neurons, indicating a necessity to control levels and timing of exogenous expression. ${ }^{17-22}$ We hypothesized that the in vivo expression patterns of Nurr1 and Foxa2 could be recapitulated in vitro by choosing appropriate expression vectors and promoters. Thus, we examined reporter GFP expression levels and timing induced by various vectors and promoters commonly used for exogenous expression induction. Because continuous expression of Nurr1 and Foxa2 is required in differentiated $\mathrm{mDA}$ neurons in adult midbrain, ${ }^{23-28}$ we examined retroviral and lentiviral vectors with diverse universal promoters known to stably induce the expression of exogenous genes.

NPCs derived from rat embryonic cortices at E14 were transduced with identical titers of the viruses expressing GFP and then differentiated the next day of transduction. In the cultured NPCs, induction of the exogenous expression driven by the retroviral pLTR was the fastest and the most efficient. Prominent GFP expression was detected from the day following transduction (Figures 1a and b). At post-transduction day 4 (differentiation day 3, D3), strong GFP expression was detected in $>70 \%$ of total cells in the cultures transduced with the GFP-retroviruses. Gene expressions induced by the lentiviral transductions in NPCs were, in general, less efficient than those with retro-pLTR during early post-transduction days (Figures 1a and b). GFP expression levels induced by PGK, EFla and ubiquitin promoters (pPGK, pEF1a, pUb) were undetectable in the first 2 days of post transduction, but then gradually increased over the next 2-3 days. The intensity of GFP expression in individual cells (estimated by mean fluorescence intensity, MFI) driven by pEF1a, pUb or pPGK were much weaker than those driven by retroviral pLTR (Figure 1c). By contrast, exogenous gene expression levels driven by the CMV and CAG promoters (pCMV, pCAG) were relatively stronger among lentiviral transduction systems (Figure 1c).

Interestingly, strong GFP expression induced by retroviral pLTR was gradually, but greatly decreased during differentiation days 5 (D5) to D15, especially in neuronal cells expressing the neuron-specific marker TuJ1 (Figures 1d-f). In contrast, GFP expression levels induced using lentiviral transduction were well maintained during the differentiation period. Ultimately, the percentage of $\mathrm{TuJ} 1+$ neurons expressing exogenous genes $(\% \mathrm{GFP}+/ \mathrm{TuJ} 1+)$ at $\mathrm{D} 15$ was greater in lentiviral pEF1a and pUb-transduced cultures than in those transduced with retroviral pLTR (Figure 1g), although the initial expression levels in the latter were weaker.

\section{Selection of vector systems for physiologically relevant Nurr1 and Foxa2 induction}

We next examined the expression patterns of Nurr1 and Foxa2 in the developing VM. Nurr1 expression was barely detected in mouse VM at early embryonic days (data not shown and Figure 2a). ${ }^{13}$ In VM at E12, Nurrl expression was identified in the intermediate and mantle zones (IZ/MZ), but not in the 
a

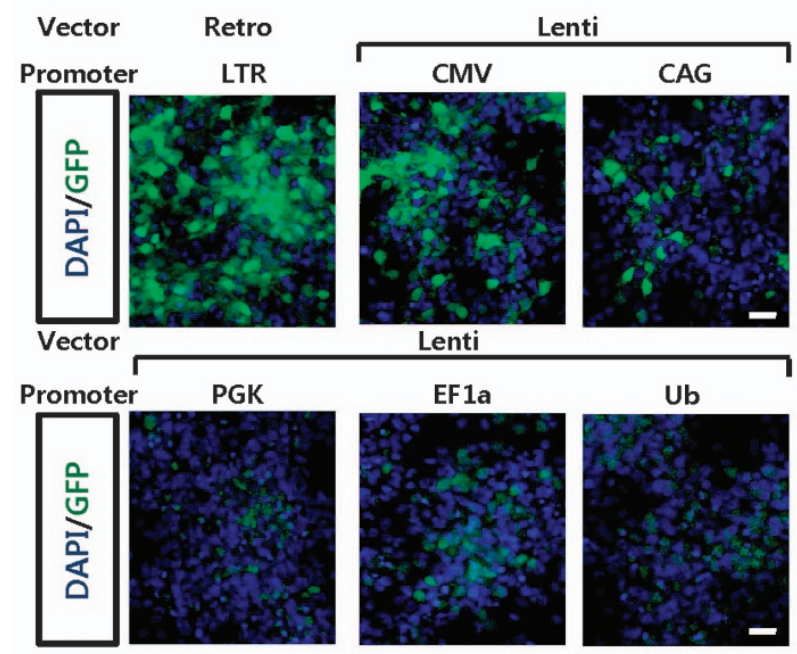

d

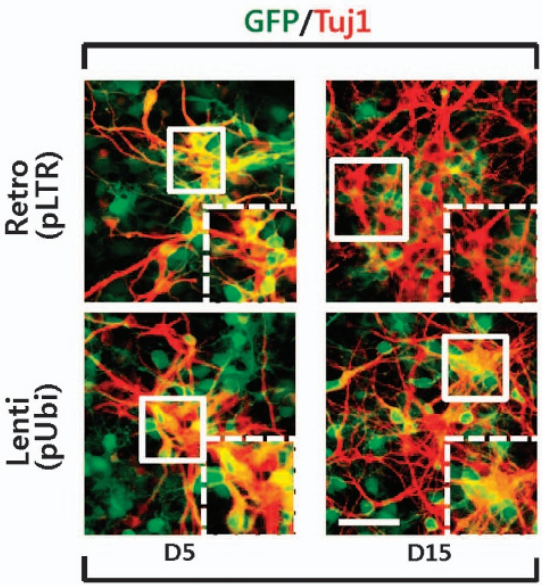

Differentiation days b
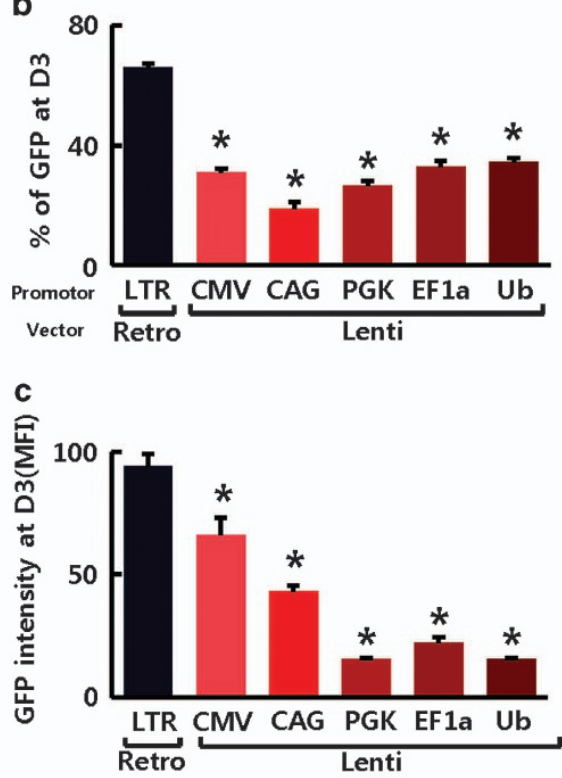
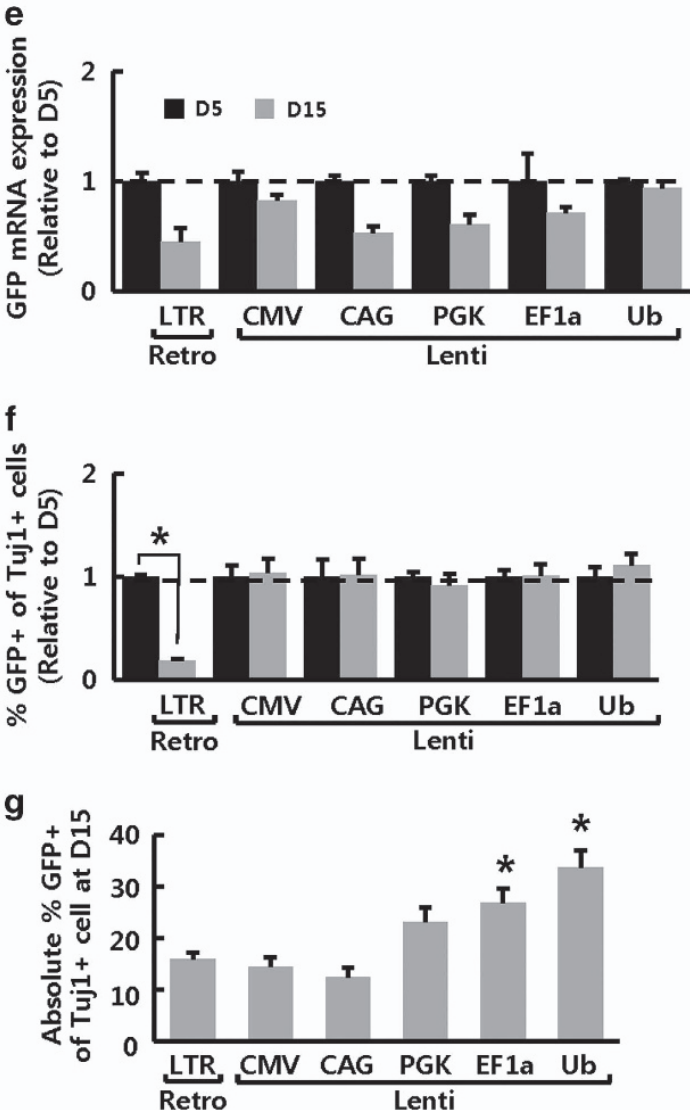

Figure 1 Exogenous expression patterns derived from retroviral and lentiviral vectors with various promoters in NPCs during differentiation in vitro. The reporter GFP gene was engineered into retroviral and lentiviral vectors with various universal promoters. NPCs derived from rat embryonic cortices at E14 were transduced with each of the GFP-expressing vectors, and the transduced NPCs were differentiated into neurons from the day following transduction. (a-c) GFP expression by various expression systems at differentiation day 3 (D3). Representative GFP+ cell images (a). Viral transduction efficiency was estimated by \% GFP+ cells of total DAPI+ cells (b) and GFP fluorescence intensity (MFI) of individual cells $(\mathbf{c})$. ( $\mathbf{d}-\mathbf{g})$ Expression of exogenous GFP during neuronal differentiation. Shown in $\mathbf{d}$ are representative GFP+/TuJ1+ cell images at D5 and D15. Insets, high-powered views of the boxed areas. Expression of GFP mRNA (e) and $\%$ of GFP+ cells out of TuJ1 cells (f) at D15 were compared with those at D5. Total numbers of TuJ1+ neurons expressing GFP at D15 (g). *Significantly different from D5 and the cultures transduced with Retro-pLTR at $P<0.05$ ( $n=20$ microscopic fields). Scale bar, $20 \mu \mathrm{m}$. DAPI, 4,6-diamidino-2-phenylindole; GFP, green fluorescence protein. 
a

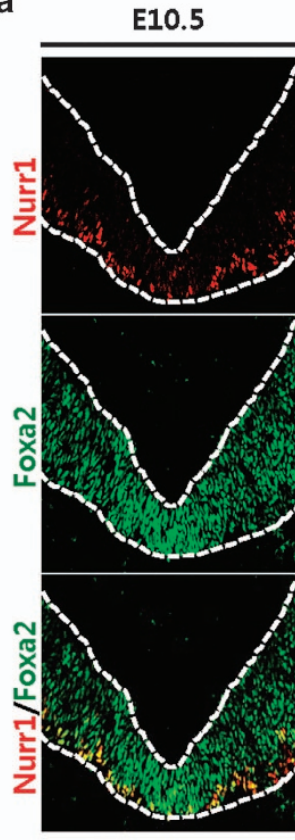

E12

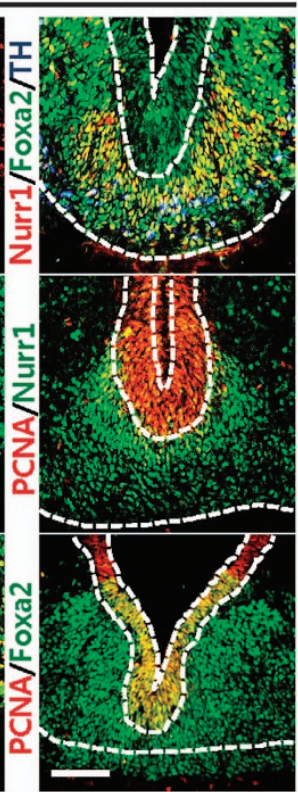

b
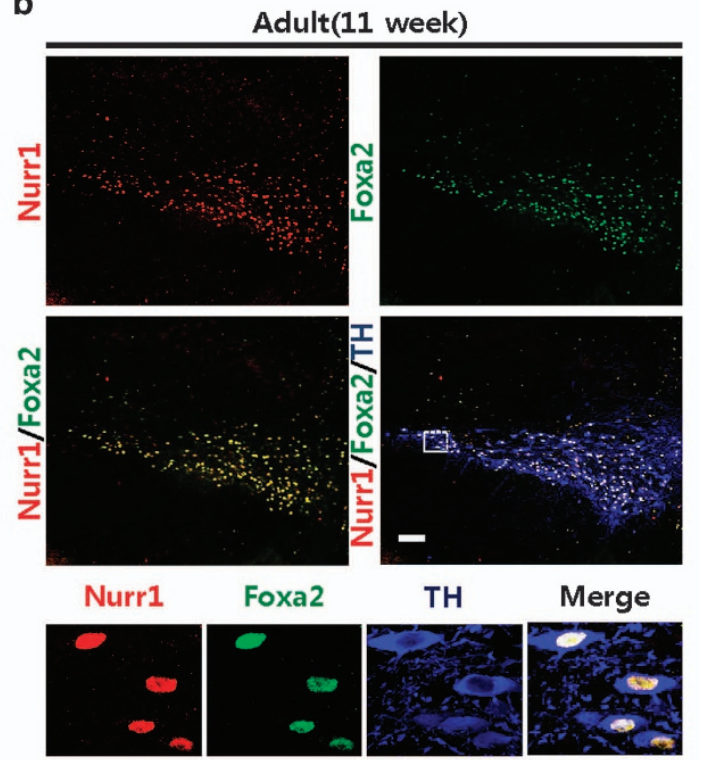

$\mathbf{C}$

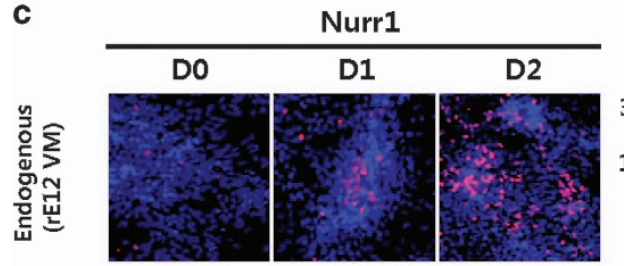

Nurr1
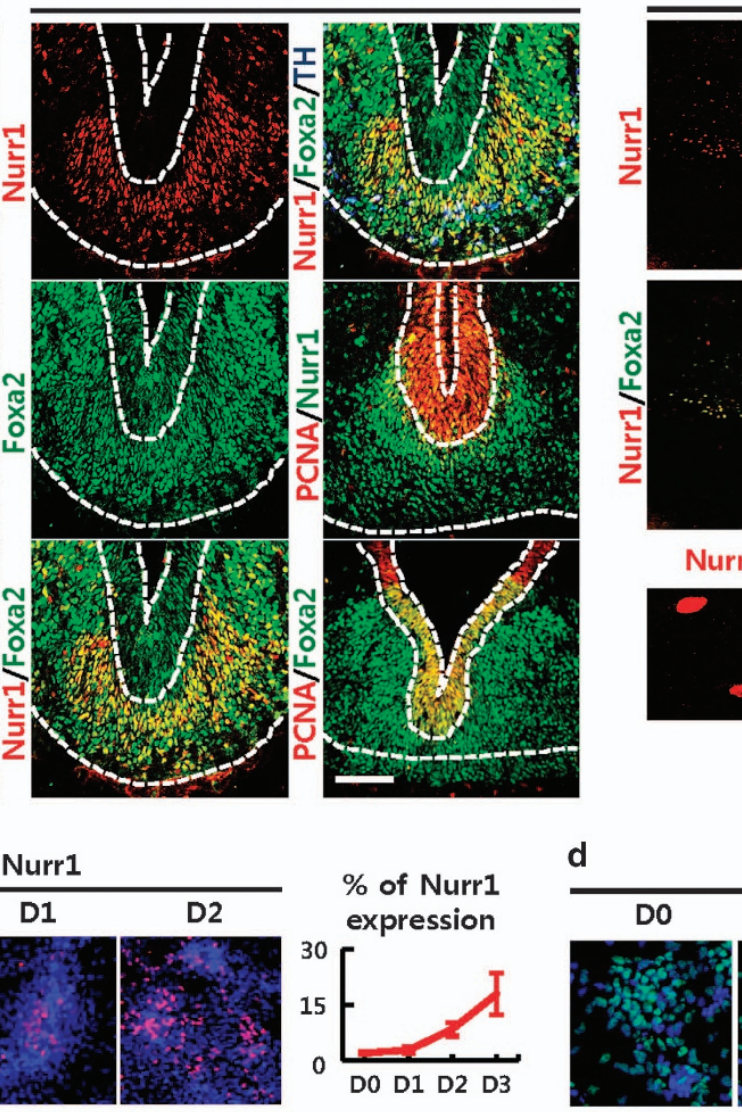

Foxa2
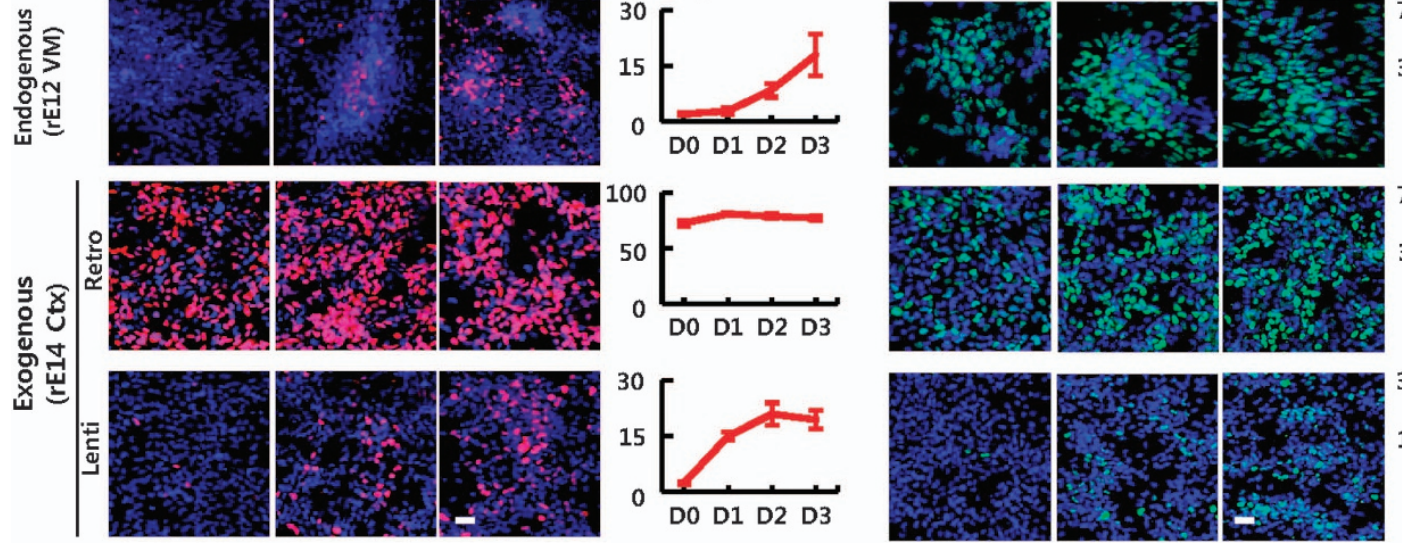

$\%$ of Foxa2 expression

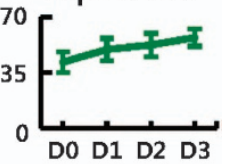

Figure 2 Endogenous Nurr1 and Foxa2 expression patterns during development are recapitulated in NPC cultures by exogenous expression driven by pUb in lentiviral transduction and pLTR in retroviral transduction. (a, b) Nurr1 and Foxa2 expression patterns in the developing VMs of mouse embryos at E10.5 and E12 (a) and SN in adult midbrain (b). Proliferating cells in VZ were labeled with PCNA (a) and DA neurons were labeled with $\mathrm{TH}(\mathbf{a}, \mathbf{b})$. Shown in the bottom row of $\mathbf{b}$ are individual and merged images of Nurr1, Foxa2 and TH staining of the boxed area. Scale bar; $100 \mu \mathrm{m}$. (c, d) Exogenous Nurr1 and Foxa2 expression levels were induced in NPCs derived from the mouse embryonic cortex using transduction with Retro-pLTR vector (middle row) or Lenti-pUb vector (last row). Exogenous expression patterns were compared with those of endogenous Nurr1 and Foxa2 expressions during in vitro differentiation of NPCs derived from rat embryonic VM at E12 (upper row). Data in graphs are means \pm s.e.m.'s of \% immunoreactive cells ( $n=10$ microscopic fields). Scale bar, $20 \mu m$. DA, dopamine; LTR, long terminal repeat; PCNA, proliferating cell nuclear antigen; SN, substantia nigra; TH, tyrosine hydroxylase.

ventricular zone (VZ), indicating commencement of Nurr1 expression from the late precursor cell stage. In contrast, Foxa2, one of the earliest genes to be expressed in the floor plate of VM, ${ }^{32,33}$ was detected in the VM at E10.5 (Figure 2a). The expression domain of Foxa2 in the VM at E12 was wider than that of Nurrl and included the alar regions lateral to the Nurr1-expressing domain (Figure 2a). Notably Foxa2 expression was detected in the proliferating VZ of E12 VM
(Figure 2a). Foxa2 expression was co-localized in Nurr1expressing cells in the IZ/MZ, and portions of Nurr1/Foxa2expressing cells in the $\mathrm{MZ}$ were positive for the DA neuronal marker, TH. In the adult midbrain, Nurr1 and Foxa2 expression were continued and co-localized in $\mathrm{TH}+\mathrm{DA}$ neurons (Figure 2b). ${ }^{23}$

In NPC cultures derived from rat embryonic VM at E12, Nurr1 expression was not detected during the proliferation 
period when the majority of cells are undifferentiated NPCs, but began to appear upon differentiation (Figure 2c). In contrast, Foxa2 expression was manifest from the proliferating VM-NPCs (Figure 2d). Forced expression of Nurr1 and Foxa2 was induced in non-dopaminergic NPC cultures (derived from cortices at E14) using lenti-pUb and retropLTR systems, and their expression patterns were compared with those of endogenous expressions. Consistent with the results presented in Figure 1, exogenous Nurr1 expression driven by lenti-pUb was delayed in a similar manner to its endogenous expression, while Foxa2 expression by retropLTR was detected early within 1 day after transduction and was similar to endogenous Foxa2 expression (Figures 2c and d). a

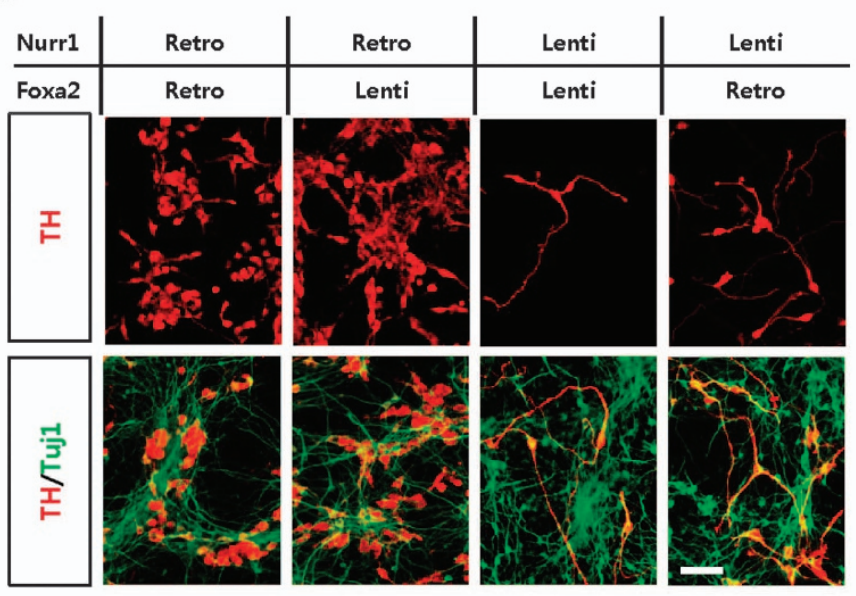

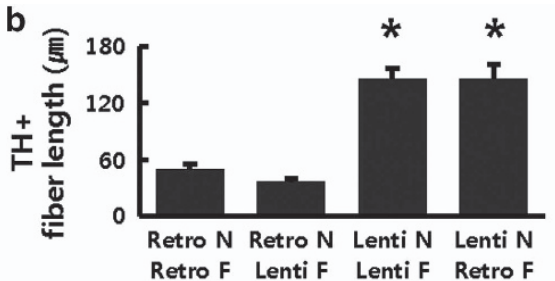

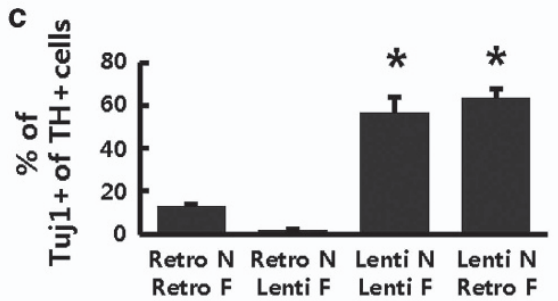

d

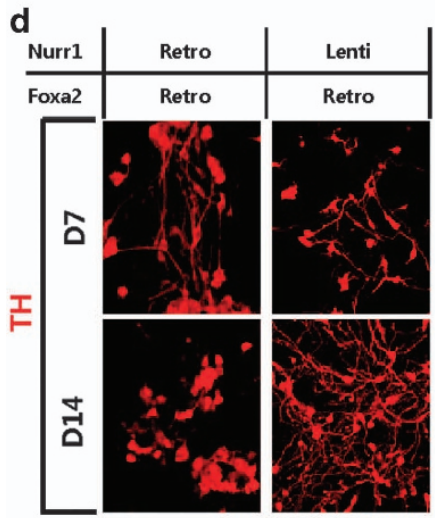

g

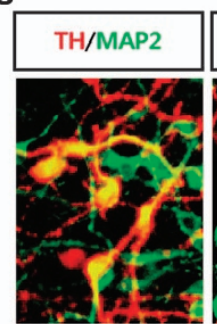

h
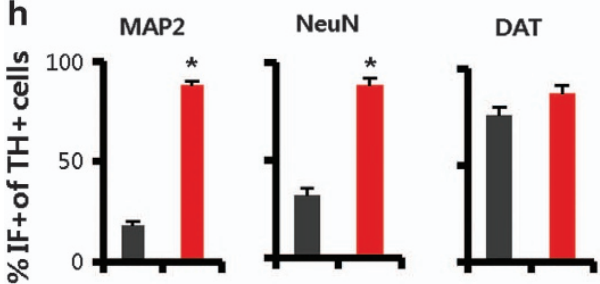

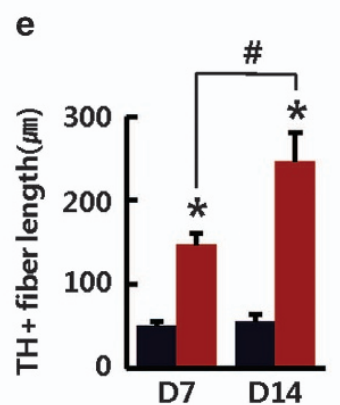

f i

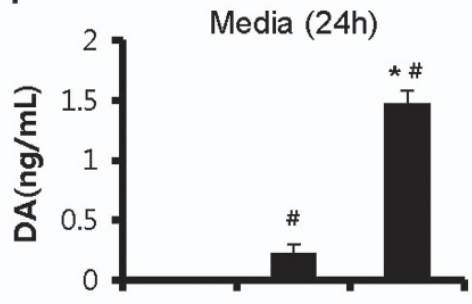

$\mathrm{KCl}$ evoked (30min)

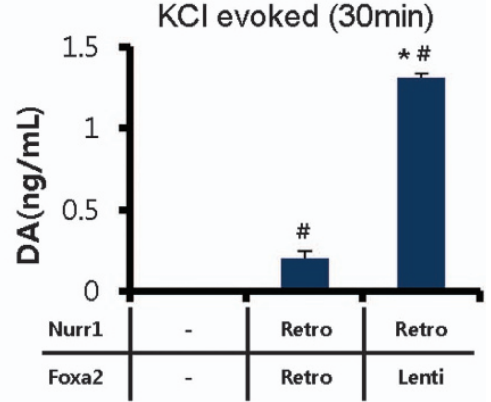


Mature midbrain-type DA neuron generation by exogenous Nurr1 and Foxa2 expression with patterns comparable to those in the developing VM

We examined whether exogenous Nurr1 and Foxa2 expression patterns really affect neuronal maturation of generated $\mathrm{TH}+$ DA cells and DA neuronal yields. NPC cultures derived from the embryonic cortices at E14 were transduced with diverse combinations of Nurr1 and Foxa2 viral vectors. None were positive for $\mathrm{TH}$ in the control cultures transduced with viruses carrying empty (retro-pLTR+lenti-pUb backbone) vectors (data not shown). Consistent with a previous study by Park et al., ${ }^{17}$ early induction of Nurr1 by retro-pLTR in NPC resulted in immature and non-neuronal DA cells (Figures 3ac). In contrast, delayed-Nurr1-expressing NPCs driven by lentipUb, which have similar developmental timing to endogenous expression, were differentiated into $\mathrm{TH} / \mathrm{TuJ} 1 \mathrm{co}$-expressing DA neurons with extensive neurite outgrowth (Figures $3 a-c$ ). Foxa2 expression timing did not affect the neuronal maturation of $\mathrm{TH}+$ cells, as both delayed and early expression of Foxa2 gave similar levels of $\mathrm{TH} / \mathrm{TuJ} 1$ co-expressing cells and neurite outgrowths. Nonetheless, recapitulation of the Foxa2 endogenous pattern (early timing and high levels) by Retro-LTR contributed to the generation of a four-fold greater $\mathrm{TH}+$ cell yield than Lenti-pUb.

In comparison with early-Foxa2/Nurr1-expressing (RetroNurr1+Retro-Foxa2) NPCs that failed to show mature TH morphology even at D14, early-Foxa2-delayed-Nurr1-expressing (Lenti-Nurr1+Retro-Foxa2) NPCs displayed mature morphology and co-expression of $\mathrm{TH}$ with Tuj1 as early as D7, and the neuronal maturation indices increased at D14 (Figures 3d-f). TH+ DA neurons acquired based on the physiological pattern of exogenous Nurr1 and Foxa2 also expressed specific markers of mature neurons (MAP2, NeuN), mature DA neurons (DAT, VMAT2), and midbrain-specific DA neurons (PITX3) at D14 (Figures $3 g$ and $h$ ). In addition, to test the functionality of the transduced midbrain-type DA neurons, we measured released DA from each differentiated population (control, Retro-Nurr1+Retro-Foxa2 and LentiNurr1+Retro-Foxa2). DA was almost undetected in the negative control cultures transduced with control viruses carrying empty vectors. As expected, much higher DA level was detected in the Lenti-Nurr1+Retro-Foxa2 transduced neurons, compared with that of Retro-Nurr1+Retro-Foxa2-transduced: $\mathrm{DA}$ releases evoked by $\mathrm{KCl}$-mediated depolarization were 1.31 (Lenti-Nurr1+Retro-Foxa2) vs 0.20 (Retro-Nurr1+RetroFoxa2) vs $0.0020 \mathrm{ng} \mathrm{ml}^{-1}$ (control; Figure 3i). Based on these findings, we concluded that the combination of LentipUb-driven Nurr1 (Lenti-Nurr1) and retro-pLTR-driven Foxa2 (Retro-Foxa2) made it possible for non-midbrain NPCs to generate mature and functional midbrain-type DA neurons.

Activation of intracellular cAMP-pCREB signaling improves midbrain-type DA neuron yield, maintenance and survival Although mature DA neurons were generated by physiological patterns of Nurr1 and Foxa2 exogenous expression, the percentage of DA neuron yield was low ( $<1 \%$ of total cells), and thus further improvement was required. Intracellular signaling of cAMP-phosphorylated cAMP response element binding protein ( $\mathrm{pCREB}$ ) has been reported to be important not only for DA neuron differentiation, but also for survival and presynaptic functionality of DA neurons. ${ }^{34-38}$ Activation of the cAMP-pCREB signal by treatment with cAMP or transduction with a constitutive active form of PKA (ca-PKA), the enzyme catalyzing phosphorylation (activation) of CREB, in NPCs transduced with Lenti-Nurr1 and Retro-Foxa2 greatly enhanced DA neuron yields ( $0.6 \%$ (untreated control) vs $9.9 \%$ (cAMP) vs $8.6 \%$ (ca-PKA), Figures $4 \mathrm{a}$ and b). Considering that Foxa2 was continuously expressed in adult mDA neurons (Figure 2b), silencing of Retro-LTR-induced expression of Foxa2 by cellular silencing machinery in long-term differentiation was another obstacle in this strategy (Figure $4 \mathrm{c}$ and also see Figure 1). However, treatment with cAMP or ca-PKA greatly prevented Foxa2 silencing (Figure $4 \mathrm{c}$ and data not shown). In addition, consistent to intracellular cAMP-pCREB roles to promote $\mathrm{mDA}$ neuronal survial, ${ }^{28,38,39}$ cAMP treatment provided the DA neurons with resistance against $\mathrm{H}_{2} \mathrm{O}_{2}$ mediated oxidative stress (Figure $4 \mathrm{~d}$ ). These findings collectively indicate that cAMP-pCREB signal activation is useful in the Nurr1+Foxa2 strategy not only to enhance DA neuronal yield and the stability of the exogene expression required for

Figure 3 Neuronal maturation and functionality of midbrain-type DA neurons generated by combinatory exogenous Nurr1 and Foxa2 expression. TH+ DA cells were generated from E14 cortical NPCs by combinatory expression of Nurr1+ Foxa2 using diverse retro-and lentiviral transductions. (a-c) Neuronal maturation of $\mathrm{TH}+$ cells. Representative immunostaining images for $\mathrm{TH}+(\mathrm{upper})$ and $\mathrm{TH}+/ \mathrm{TuJ} 1+$ cells (lower) (a). Neuronal maturity of TH+ DA cells was evaluated by $\mathrm{TH}+$ fiber length (b) and \% TuJ1+ cells out of total TH+ cells (c) at D7. *Significantly different from cultures transduced with Retro-pLTR-Nurr1+Retro-pLTR-Foxa2 at $P<0.05, n=50$ TH+ cells (b), 20 microscopic fields $(\mathbf{c})$. (d-f) Neuronal maturation of $\mathrm{TH}+$ cells during the late differentiation period (D7-D14). Representative images for $\mathrm{TH}+$ cells (left), TH+/Tuj1+ (right upper) and TH+/MAP2+ cells (right lower) at differentiation days 7 and 14 (D7 and D14) (d). TH+ fiber length (e) and co-expression of the TH/Tuj1 (f) at D7 and D14. Significantly different from cultures transduced with Retro-pLTR-Nurr1 +Retro-pLTR-Foxa2* and from D7\# at $P<0.05, n=50 \mathrm{TH}+$ cells (e), 20 microscopic fields (f). (g-i) Phenotypic and functional analysis of $\mathrm{TH}+\mathrm{mDA}$ neuronal cells differentiated from NPCs transduced with Lenti-pUb-Nurr1+Retro-pLTR-Foxa2. Mature midbrain DA neuron phenotypes were analyzed by co-expression of neuronal (MAP2 and NeuN), general DA neuronal (DAT, VMAT2) and midbrain-specific DA neuronal marker (PITX3) (g, h). Measurement of released DA in media (24 h; upper) and evoked by $\mathrm{KCl}$ ( $56 \mathrm{~mm}$; $30 \mathrm{~min}$; lower) from differentiated neurons (i). *Significantly different from the cultures transduced with Retro-pLTR-Nurr1+Retro-pLTR-Foxa2 at $P<0.05$, $n=20$ microscopic fields (h) and three independent cultures (i). \#Significantly different from the cultures transduced with control vector at $P<0.05$. Scale bar, $20 \mu \mathrm{m}$. DA, dopamine; TH, tyrosine hydroxylase. 

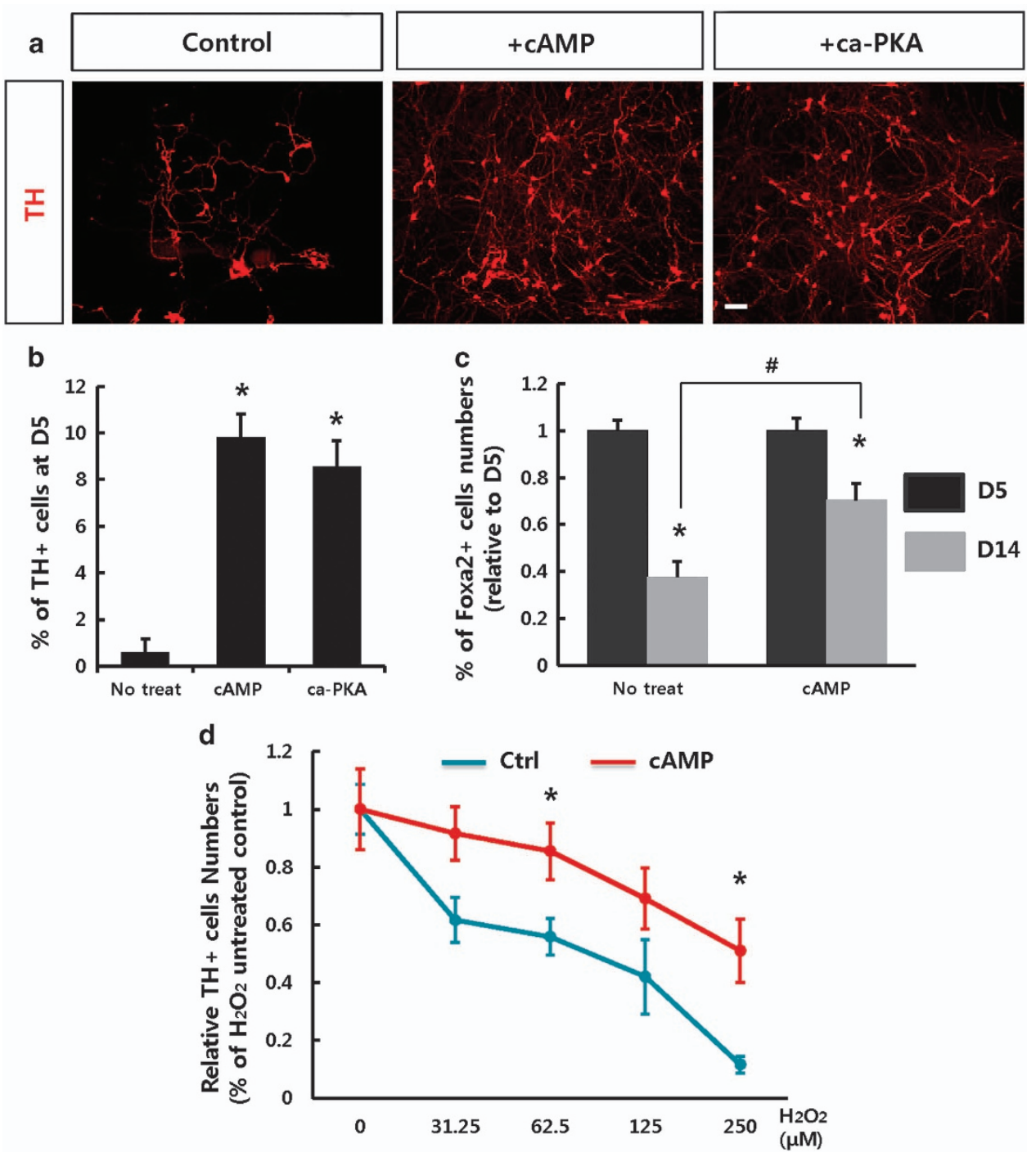

Figure 4 cAMP-pCREB signal activation promotes differentiation, survival and exogenous expression maintenance of midbrain-type DA neuron. The cAMP-pCREB signal was activated in Nurr1(Lenti-pUb)+Foxa2(Retro-pLTR)-transduced NPCs by treatment of cAMP (0.5 mM) or co-transduction with ca-PKA (using lenti-pUb vector). (a, b) Effect of cAMP-pCREB signal activation on Nurr1+Foxa2-induced DA neuronal yield. Shown in a are representative images for TH+ cells at D5 in the indicated cultures. Scale bar, $20 \mu \mathrm{m}$. DA cell yields estimated by \%TH+ cells (b). *Significantly different from the untreated control at $P<0.05$. (c) Effect of cAMP-pCREB signal activation on maintenance of exogenous Retro-pLTR-Foxa2 expression during late differentiation period (D5-D14). Significantly different from D5* $^{*}$ and from cAMP-untreated ${ }^{\#}$ at $P<0.05$. (d) $\mathrm{TH}+\mathrm{DA}$ neurons treated with cAMP showed higher cell viability in the presence of $\mathrm{H}_{2} \mathrm{O}_{2}-$ mediated toxic stimuli. $\mathrm{TH}+$ cell generated by Nurr1+Foxa2 expression (at D13) were treated with various doses of $\mathrm{H}_{2} \mathrm{O}_{2}$ in the absence or presence of cAMP. Statistical analyses were used to compare cAMP-treated and untreated control values at each $\mathrm{H}_{2} \mathrm{O}_{2}$ dose. ${ }^{*} P<0.05$ $(n=5)$. cAMP, cyclic AMP; DA, dopamine; NPCs, neural stem/precursor cells; TH, tyrosine hydroxylase.

DA phenotype maintenance, but also in order to promote DA neuron resistance against toxic stimuli.

Next, based on in vitro observations, intra-striatal transplantation was carried out with NPCs transduced with a combination of Lenti-Nurr1+Retro-Foxa2+Lenti-caPKA into PD model rats. Histological assessment 4 weeks after transplantation demonstrated that transduced NPCs generated TH+ DA neurons with an extremely mature morphology, which extended their neurite outgrowth towards the host striatum (Figure 5). However, unfortunately, the in vivo functionality of the transplanted cells could not be observed in the analysis of
PD-related behaviors in a series of transplantation experiments, mainly due to poor donor cell engraftment with few $\mathrm{TH}+$ cells in the grafts $(<100$ cells). This indicates the necessity of further transplantation studies with improved donor cell engraftment. Although the in vivo functionality of the Nurr1+Foxa2+caPKA-NPCs was not proven in this study, this study is useful because it describes a method for generating mature and functional midbrain-type DA neurons for use in in vitro bioassays aimed at therapeutic developments related to $\mathrm{PD}$ or other disorders associated with midbrain-type DA neurons. 


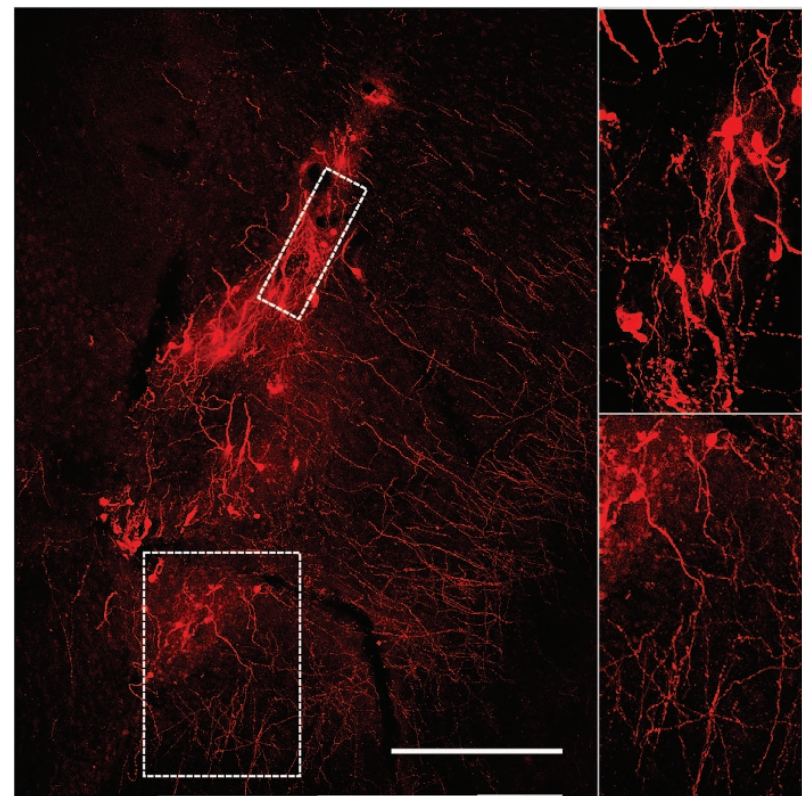

Figure $5 \mathrm{TH}+$ cell graft formed by transplantation with NPCs transduced with Lenti-pUb-Nurr1+Retro-pLTR-Foxa2+Lenti-pUb-caPKA. Cortical NPCs transduced with Lenti-pUb-Nurr1+Retro-pLTRFoxa2+Lenti-pUb-ca-PKA were transplanted into the striatum of a PD model rat. The brain was cryosectioned and stained with $\mathrm{TH}$, and confocal images were taken at 4 weeks after transplantation. $\mathrm{TH}+$ cells in the boxed areas were enlarged in the right panels. Scale bar, $100 \mu \mathrm{m}$. NPCs, neural stem/precursor cells; TH, tyrosine hydroxylase.

\section{DISCUSSION}

Midbrain-type DA neuron differentiation is a prerequisite for stem cell-based therapeutic developments related to PD and disorders associated with $\mathrm{mDA}$ neurons; however, efficient mDA neuron differentiation from cultured stem cells is challenging. To overcome these difficulties, we utilized forced expression of Nurr1 and Foxa2, the combinatory action of which facilitates acquisition of general DA and midbrain DA neuron phenotypes at later stages of midbrain development. ${ }^{9,16}$ However, simple overexpression of these factors frequently yields non-neuronal and immature DA neurons. ${ }^{17-22} \mathrm{We}$ sought to overcome the immaturity problem by replicating the physiologic expression patterns of the developmental factors in the forced expression strategy. Using appropriate expression vector systems such as Lenti-pUb for Nurr1 and Retro-pLTR for Foxa2 expression, we successfully generated midbrain-type DA neurons with an appropriate morphology and stage of maturity.

Another critical issue is stable exogene expression in differentiated mDA neurons, since expression of Nurr1 and Foxa 2 continues in $\mathrm{mDA}$ neurons in the adult midbrain and is critical for phenotype maintenance, function and survival in this neuronal subtype. ${ }^{23-28}$ However, we observed that Retro-pLTR-mediated Foxa2 expression is silenced after long-term differentiation, although theoretically, retroviral vector-mediated expression should be stable. It has been consistently reported that during hematopoietic differentiation of mouse ESCs, gene expression from the retrovirus LTR, but not from the lentivirus LTR, is severely reduced ${ }^{40}$; this appears to be caused by DNA methylation of the retroviral LTR promoter. ${ }^{41}$ We found that silencing of the Retro-LTR promoter during neuronal differentiation is substantially prevented by cAMP treatment or caPKA expression. It is likely direct binding of pCREB activated by cAMP (caPKA) to the LTR promoter that results in this activation, based on the existence of consensus CREB-binding sequences in the LTR promoter and the positive regulatory role of CREB binding in promoter activity. In addition, cAMP-pCREB activation in Nurr1+Foxa2-transduced NPCs promoted mDA neuronal yield and survival/resistance of the resulting $\mathrm{mDA}$ neurons against toxic insult.

On the basis of the in vitro findings, we performed in vivo functional assays by transplanting the Nurr1+Foxa2+caPKAtransduced NPCs into PD model rats. The success of cell transplantation-mediated PD therapy depends on efficient mDA neuron engraftment and neuronal maturation of the engrafted $\mathrm{mDA}$ neurons. Neuronal maturation leading to synaptic connections between grafted $\mathrm{mDA}$ neurons and host neurons is critical for long-term therapeutic effects. ${ }^{42,43}$ In histological assessment, TH+ DA neurons engrafted in the host striatum exhibited an extremely mature morphology, with their long neurite outgrowths extending toward the host striatum. However, unfortunately, the in vivo functionality of the transplanted cells could not be observed in the analysis of PD-related behaviors in a series of transplantation experiments, mainly due to lack of sufficient engraftment of TH+ cells. Poor donor cell engraftment was probably due to the cell toxicity induced by multiple gene transductions during donor cell preparation. We noticed that viral transduction was somewhat toxic to cultured NPCs. Thus, donor NPCs transduced with multiple genes are prone to cell death in the hostile in vivo host environment after transplantation, although the transduced NPCs can survive and differentiate into mature TH+ DA neurons under in vitro culture conditions supplemented with sufficient trophic factors. In addition, both Nurr1 and Foxa2 are strong factors involved in cell cycle arrest. ${ }^{9,44,45}$ Thus, the cell arrest effects of the transgenes are thought to contribute to poor $\mathrm{TH}+$ cell engraftment, as the proliferative capacity of donor cells is also beneficial for their engraftment and to the formation of a sound graft of the transplanted tissue.

In conclusion, this study illustrates the utility of artificial cell fate specification with controlled gene expression, which can replicate the gene profile of actual brain development. The data indicate that sequential and controlled expression levels of transcription factors Foxa2 and Nurr1 along with cAMPpCREB signal activation result in fully mature midbrain-type DA neurons with stable phenotype maintenance and improved cell survival. Future studies are needed to establish the usefulness of this strategy in NPCs of human origin and to produce successful engraftment and therapeutic efficacy in PD.

\section{CONFLICT OF INTEREST}

The authors declare no conflict of interest. 


\section{ACKNOWLEDGEMENTS}

This work was supported by a grant from the Medical Research Center (2008-0062287), funded by the National Research Foundation of Korea (NRF) of the Ministry of Science, ICT and Future Planning, Republic of Korea to S-HL and by the Basic Science Research Program (NRF-2014R1A1A2057443) through the NRF funded by the Ministry of Education to J-wS.

1 Lozano AM, Lang AE, Hutchison WD, Dostrovsky JO. New developments in understanding the etiology of Parkinson's disease and in its treatment. Curr Opin Neurobiol 1998; 8: 783-790.

2 Barzilai A, Melamed E. Molecular mechanisms of selective dopaminergic neuronal death in Parkinson's disease. Trends Mol Med 2003; 9: $126-132$.

3 Olanow CW, Kordower JH, Freeman TB. Fetal nigral transplantation as a therapy for Parkinson's disease. Trends Neurosci 1996; 19: 102-109.

4 Piccini P, Brooks DJ, Bjorklund A, Gunn RN, Grasby PM, Rimoldi 0 et al. Dopamine release from nigral transplants visualized in vivo in a Parkinson's patient. Nat Neurosci 1999; 2: 1137-1140.

5 Deierborg T, Soulet D, Roybon L, Hall V, Brundin P. Emerging restorative treatments for Parkinson's disease. Prog Neurobiol 2008; 85: 407-432.

6 Lee JY, Chang MY, Park CH, Kim HY, Kim JH, Son H et al. Ascorbateinduced differentiation of embryonic cortical precursors into neurons and astrocytes. J Neurosci Res 2003; 73: 156-165.

7 Yan J, Studer L, McKay RD. Ascorbic acid increases the yield of dopaminergic neurons derived from basic fibroblast growth factor expanded mesencephalic precursors. J Neurochem 2001; 76: 307-311.

8 Metzakopian E, Lin W, Salmon-Divon M, Dvinge H, Andersson E, Ericson J et al. Genome-wide characterization of Foxa2 targets reveals upregulation of floor plate genes and repression of ventrolateral genes in midbrain dopaminergic progenitors. Development 2012; 139: 2625-2634.

9 Lee HS, Bae EJ, Yi SH, Shim JW, Jo AY, Kang JS et al. Foxa2 and Nurr1 synergistically yield A9 nigral dopamine neurons exhibiting improved differentiation, function, and cell survival. Stem Cells 2010; 28: 501-512.

10 Ang SL. Foxa1 and Foxa2 transcription factors regulate differentiation of midbrain dopaminergic neurons. Adv Exp Med Biol 2009; 651: 58-65.

11 Ferri AL, Lin W, Mavromatakis YE, Wang JC, Sasaki H, Whitsett JA et al. Foxa1 and Foxa2 regulate multiple phases of midbrain dopaminergic neuron development in a dosage-dependent manner. Development 2007; 134: 2761-2769.

12 Kittappa R, Chang WW, Awatramani RB, McKay RD. The foxa2 gene controls the birth and spontaneous degeneration of dopamine neurons in old age. PLoS Biol 2007; 5: e325.

13 Zetterstrom RH, Solomin L, Jansson L, Hoffer BJ, Olson L, Perlmann T. Dopamine neuron agenesis in Nurr1-deficient mice. Science 1997; 276: 248-250.

14 Castillo SO, Baffi JS, Palkovits M, Goldstein DS, Kopin IJ, Witta J et al. Dopamine biosynthesis is selectively abolished in substantia nigra/ ventral tegmental area but not in hypothalamic neurons in mice with targeted disruption of the Nurr1 gene. Mol Cell Neurosci 1998; 11: 36-46.

15 Saucedo-Cardenas O, Quintana-Hau JD, Le WD, Smidt MP, Cox JJ, De Mayo $\mathrm{F}$ et al. Nurr1 is essential for the induction of the dopaminergic phenotype and the survival of ventral mesencephalic late dopaminergic precursor neurons. Proc Natl Acad Sci USA 1998; 95: 4013-4018.

16 Yi SH, He XB, Rhee YH, Park CH, Takizawa T, Nakashima K et al. Foxa2 acts as a co-activator potentiating expression of the Nurr1-induced DA phenotype via epigenetic regulation. Development 2014; 141: 761-772.

17 Park CH, Lim MS, Rhee YH, Yi SH, Kim BK, Shim JW et al. In vitro generation of mature dopamine neurons by decreasing and delaying the expression of exogenous Nurr1. Development 2012; 139: 2447-2451.

18 Kim JY, Koh HC, Lee JY, Chang MY, Kim YC, Chung HY et al. Dopaminergic neuronal differentiation from rat embryonic neural precursors by Nurr1 overexpression. J Neurochem 2003; 85: 1443-1454.
19 Sakurada K, Ohshima-Sakurada M, Palmer TD, Gage FH. Nurr1, an orphan nuclear receptor, is a transcriptional activator of endogenous tyrosine hydroxylase in neural progenitor cells derived from the adult brain. Development 1999; 126: 4017-4026.

20 Park CH, Kang JS, Kim JS, Chung S, Koh JY, Yoon EH et al. Differential actions of the proneural genes encoding Mash1 and neurogenins in Nurr1induced dopamine neuron differentiation. J Cell Sci 2006; 119: 2310-2320.

21 Kim HJ, Sugimori M, Nakafuku M, Svendsen CN. Control of neurogenesis and tyrosine hydroxylase expression in neural progenitor cells through bHLH proteins and Nurr1. Exp Neurol 2007; 203: 394-405.

22 Sonntag KC, Simantov R, Kim KS, Isacson O. Temporally induced Nurr1 can induce a non-neuronal dopaminergic cell type in embryonic stem cell differentiation. Eur J Neurosci 2004; 19: 1141-1152.

23 Oh SM, Chang MY, Song JJ, Rhee YH, Joe EH, Lee HS et al. Combined Nurr1 and Foxa2 roles in the therapy of Parkinson's disease. EMBO Mol Med 2015; 7: 510-525.

24 Volakakis N, Kadkhodaei B, Joodmardi E, Wallis K, Panman L, Silvaggi J et al. NR4A orphan nuclear receptors as mediators of CREBdependent neuroprotection. Proc Natl Acad Sci USA 2010; 107: 12317-12322.

25 Malewicz M, Kadkhodaei B, Kee N, Volakakis N, Hellman U, Viktorsson K et al. Essential role for DNA-PK-mediated phosphorylation of NR4A nuclear orphan receptors in DNA double-strand break repair. Genes Dev 2011; 25: 2031-2040.

26 Decressac M, Kadkhodaei B, Mattsson B, Laguna A, Perlmann T, Bjorklund A. alpha-Synuclein-induced down-regulation of Nurr1 disrupts GDNF signaling in nigral dopamine neurons. Sci Trans/ Med 2012; 4: 163 ra56.

27 Kadkhodaei B, Alvarsson A, Schintu N, Ramskold D, Volakakis N, Joodmardi $E$ et al. Transcription factor Nurrl maintains fiber integrity and nuclear-encoded mitochondrial gene expression in dopamine neurons. Proc Natl Acad Sci USA 2013; 110: 2360-2365.

28 Stott SR, Metzakopian E, Lin W, Kaestner KH, Hen R, Ang SL. Foxa1 and foxa2 are required for the maintenance of dopaminergic properties in ventral midbrain neurons at late embryonic stages. J Neurosci 2013; 33: 8022-8034.

29 Johe KK, Hazel TG, Muller T, Dugich-Djordjevic MM, McKay RD. Single factors direct the differentiation of stem cells from the fetal and adult central nervous system. Genes Dev 1996; 10: 3129-3140.

30 Studer L, Tabar V, McKay RD. Transplantation of expanded mesencephalic precursors leads to recovery in parkinsonian rats. Nat Neurosci 1998; 1: 290-295.

31 Bae EJ, Lee HS, Park CH, Lee SH. Orphan nuclear receptor Nurr1 induces neuron differentiation from embryonic cortical precursor cells via an extrinsic paracrine mechanism. FEBS Lett 2009; 583: $1505-1510$

32 Ang SL, Wierda A, Wong D, Stevens KA, Cascio S, Rossant J et al. The formation and maintenance of the definitive endoderm lineage in the mouse: involvement of HNF3/forkhead proteins. Development 1993; 119: 1301-1315.

33 Lin W, Metzakopian E, Mavromatakis YE, Gao N, Balaskas N, Sasaki H et al. Foxa1 and Foxa2 function both upstream of and cooperatively with $L m x 1 a$ and $L m x 1 b$ in a feedforward loop promoting mesodiencephalic dopaminergic neuron development. Dev Biol 2009; 333: 386-396.

34 Ko JY, Lee HS, Park CH, Koh HC, Lee YS, Lee SH. Conditions for tumorfree and dopamine neuron-enriched grafts after transplanting human ES cell-derived neural precursor cells. Mol Ther 2009; 17: 1761-1770.

35 Kumer SC, Vrana KE. Intricate regulation of tyrosine hydroxylase activity and gene expression. J Neurochem 1996; 67: 443-462.

36 Leviel V, Guibert B, Mallet J, Faucon-Biguet N. Induction of tyrosine hydroxylase in the rat substantia nigra by local injection of forskolin. J Neurosci Res 1991; 30: 427-432.

37 Barneda-Zahonero B, Servitja JM, Badiola N, Minano-Molina AJ, Fado R, Saura CA et al. Nurr1 protein is required for N-methyl-D-aspartic acid (NMDA) receptor-mediated neuronal survival. J Biol Chem 2012; 287: 11351-11362.

38 Mourlevat S, Troadec JD, Ruberg M, Michel PP. Prevention of dopaminergic neuronal death by cyclic AMP in mixed neuronal/glial mesencephalic cultures requires the repression of presumptive astrocytes. Mol Pharmacol 2003; 64: 578-586.

39 Hulley $P$, Hartikka J, Lubbert $H$. Cyclic AMP promotes the survival of dopaminergic neurons in vitro and protects them from the toxic effects of MPP+. J Neural Transm Suppl 1995; 46: 217-228. 
40 Hamaguchi I, Woods NB, Panagopoulos I, Andersson E, Mikkola H, Fahlman $\mathrm{C}$ et al. Lentivirus vector gene expression during ES cell-derived hematopoietic development in vitro. J Virol 2000; 74: 10778-10784.

41 Laker C, Meyer J, Schopen A, Friel J, Heberlein C, Ostertag W et al. Host cis-mediated extinction of a retrovirus permissive for expression in embryonal stem cells during differentiation. J Virol 1998; 72: 339-348.

42 Tonnesen J, Kokaia M. Electrophysiological investigations of synaptic connectivity between host and graft neurons. Prog Brain Res 2012; 200 . 97-112.

43 Stroemer RP, Kent TA, Hulsebosch CE. Neocortical neural sprouting, synaptogenesis, and behavioral recovery after neocortical infarction in rats. Stroke 1995; 26: 2135-2144.

44 Castro DS, Hermanson E, Joseph B, Wallen A, Aarnisalo P, Heller A et al. Induction of cell cycle arrest and morphological differentiation by Nurr1 and retinoids in dopamine MN9D cells. J Biol Chem 2001; 276: 43277-43284.
45 Liu M, Lee DF, Chen CT, Yen CJ, Li LY, Lee HJ et al. IKKalpha activation of NOTCH links tumorigenesis via FOXA2 suppression. Mol Cell 2012; 45 171-184.

(c) (i)(-) (2) This work is licensed under a Creative Commons Attribution-NonCommercial-ShareAlike

International License. The images or other third party material in this article are included in the article's Creative Commons license, unless indicated otherwise in the credit line; if the material is not included under the Creative Commons license, users will need to obtain permission from the license holder to reproduce the material. To view a copy of this license, visit http://creativecommons.org/licenses/by-nc-sa/4.0/ 